\title{
Remote ischaemic conditioning for stroke: unanswered questions and future directions
}

\author{
Sheharyar Baig, ${ }^{1}$ Bethany Moyle, ${ }^{1}$ Krishnan Padmakumari Sivaraman Nair, ${ }^{2}$ \\ Jessica Redgrave, ${ }^{1}$ Arshad Majid, ${ }^{3}$ Ali Ali (D) ${ }^{4,5}$
}

To cite: Baig S, Moyle B, Nair KPS, et al. Remote ischaemic conditioning for stroke: unanswered questions and future directions. Stroke \& Vascular Neurology 2021;6: e000722. doi:10.1136/svn2020-000722

- Prepublication history and additional material is published online only. To view please visit the journal online (http://dx.doi. org/10.1136/svn-2020-000722).

Received 29 0ctober 2020 Revised 13 January 2021 Accepted 31 January 2021 Published Online First 26 April 2021

Check for updates

(C) Author(s) (or their employer(s)) 2021. Re-use permitted under CC BY-NC. No commercial re-use. See rights and permissions. Published by BMJ.

${ }^{1}$ Cerebrovascular Medicine, The University of Sheffield Institute for Translational Neuroscience, Sheffield, UK

${ }^{2}$ Neurosciences, Sheffield

Teaching Hospitals NHS

Foundation Trust, Sheffield, UK

${ }^{3}$ Faculty of Medicine and

Dentistry, University of Sheffield,

Sheffield, UK

${ }^{4}$ Geriatrics and Stroke Medicine, Sheffield Teaching Hospitals NHS Foundation Trust, Sheffield, UK

${ }^{5}$ Sheffield NIHR Biomedical

Research Centre, The University of Sheffield, Sheffield, UK

Correspondence to

DrAli Ali; ali.ali@sheffield.ac.uk

\section{ABSTRACT}

Remote ischaemic conditioning (RIC) refers to a process whereby periods of intermittent ischaemia, typically via the cyclical application of a blood pressure cuff to a limb at above systolic pressure, confers systemic protection against ischaemia in spatially distinct vascular territories. The mechanisms underlying this have not been characterised fully but have been shown to involve neural, hormonal and systemic inflammatory signalling cascades. Preclinical and early clinical studies have been promising and suggest beneficial effects of RIC in acute ischaemic stroke, symptomatic intracranial stenosis and vascular cognitive impairment. Through systematic searches of several clinical trials databases we identified 48 active clinical trials of RIC in ischaemic stroke, intracerebral haemorrhage and subarachnoid haemorrhage. We summarise the different RIC protocols and outcome measures studied in ongoing clinical trials and highlight which studies are most likely to elucidate the underlying biological mechanisms of RIC and characterise its efficacy in the near future. We discuss the uncertainties of RIC including the optimal frequency and duration of therapy, target patient groups, cost-effectiveness, the confounding impact of medications and the absence of a clinically meaningful biomarker of the conditioning response. With several large clinical trials of RIC expected to report their outcomes within the next 2 years, this review aims to highlight the most important studies and unanswered questions that will need to be addressed before this potentially widely accessible and low-cost intervention can be used in clinical practice.

\section{INTRODUCTION}

Remote ischaemic conditioning (RIC) refers to the process by which cycles of temporary ischaemia, typically through the application of a manual or electronic tourniquet to a limb at above systolic blood pressure, confers a systemic protection against future ischaemic injuries in remote vascular territories,. ${ }^{1}$ The concept of ischaemic conditioning as a protective technique was described by Murry et al who found that $5 \mathrm{~min}$ cycles of occlusion and reperfusion of canine circumflex arteries repeated 4 times $(4 \times 5$ mins $)$ prior to experimental myocardial infarction in the same arterial territory led to a $25 \%$ reduction in myocardial infarct size compared with controls. ${ }^{2}$ Subsequently brief episodes of the same ischaemic conditioning protocol applied to the circumflex artery was found to have protective properties when the experimental infarct was induced in a different arterial territory ${ }^{3}-$ RIC. It was later discovered that applying similar sublethal episodes of ischaemic to remote organs $^{4}$ or a limb, ${ }^{5}$ also offered protection to subsequent ischaemic or reperfusion injury, not only to the heart, but to other organs including liver, ${ }^{6}$ kidneys ${ }^{7}$ and brain. ${ }^{8}$ These findings have led to the investigation of its use in cerebrovascular disease.

The timing of RIC in relation to ischaemia and subsequent reperfusion defines the terms preconditioning (RIPreC), perconditioning (RIPerC) and postconditioning (RIPostC) (figure 1). The protective effect of ischaemic conditioning occurs at different time frames: the acute effect is induced immediately and lasts 2 hours while the delayed protection occurs at 12-24 hours, lasting upto 48-72 hours. ${ }^{9}$ Furthermore, repeated cycles of RIC (chronic RIC) may induce distinct adaptations that influence stroke recurrence and recovery. ${ }^{1011}$

A small number of studies have investigated the use of RIC in acute and chronic stroke, ${ }^{12-19}$ and have been summarised in recent review articles. ${ }^{20}{ }^{21}$ However, there is a rapidly evolving research landscape with multiple ongoing clinical trials that, when completed and published, will have a significant impact on understanding the utility of RIC in stroke. Here, we outline the ongoing clinical trials investigating RIC in acute and chronic stroke, expand on the key remaining research questions, comprehensively detail the remaining barriers to translation into clinical practice 


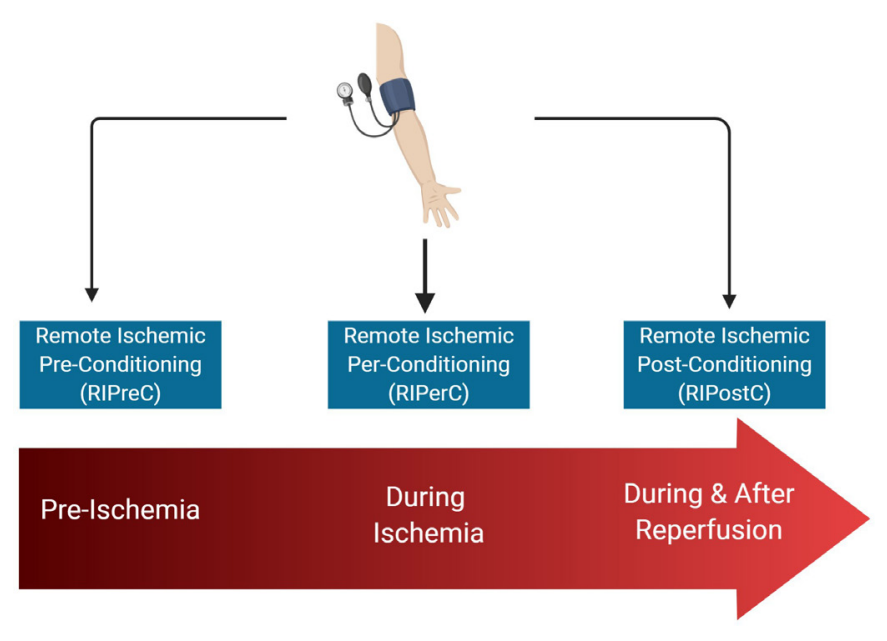

Figure 1 Model of remote ischaemic conditioning.

and make practice recommendations for future clinical trial design.

\section{METHODS}

We searched the following trials registries/journals (25 August 2020) for ongoing or completed trials of RIC in stroke:

1. ClinicalTrials.gov (https://clinicaltrials.gov/).

2. WHO International Clinical Trials Registry Platform (https://www.who.int/ictrp/search/en/).

3. ISRCTN Registry (https://www.isrctn.com/).

4. European Union Clinical Trials Register (https://www. clinicaltrialsregister.eu/).

5. BioMedCentral Trials Journal (https://trialsjournal. biomedcentral.com/).

6. Chinese Clinical Trials Registry (www.chictr.org.cn).

Search terms included 'ischaemic conditioning' and 'stroke'. In the Chinese Clinical Trials Registry 'conditioning' was used as a search term to widen results. Further studies were identified from previous published articles and citation searches.

Active studies were included if the RIC intervention (cycles of temporary upper or lower limb ischaemia) was being studied in patients with stroke (ischaemic or haemorrhagic stroke), transient ischaemic attack or subarachnoid haemorrhage. Completed studies were included if they met the search criteria above but had not yet published the full results.

Studies were labelled as having an 'Unknown' status if: (1) the study status was not available on the relevant clinical trials registry or (2) more than 2 years elapsed since the estimated completion date, without publication of results or trial registry update and (3) no email correspondence was received from chief investigator of the study.

\section{Relevant studies}

We identified 48 studies investigating RIC in stroke (online supplemental material). After analysing the trial protocols for each study, we determined that the RIC intervention is being studied in three distinct time frames:

1. RIPreC-the administration of RIC prior to a surgical or endovascular procedure where there is a significant risk of stroke.

2. RIPerC and/or early remote ischaemic postconditioning (Early RIPostC) - the administration of RIC in the hours to days after the onset of an acute stroke (ischaemic, haemorrhagic or subarachnoid haemorrhage)

3. Chronic remote RIPostC (Chronic RIC) - the administration of repeated cycles of RIC for weeks to months in patients with a history of stroke.

Table 1 summarises the variety of populations, RIC protocols, outcome measures and estimated completion dates of ongoing clinical trials in RIC. A more detailed summary of individual studies can be found in online supplemental material.

RIPreC: The use of RIPreC is a clinical application of the classic paradigm of ischaemic preconditioning whereby a period of temporary ischaemia induces protective changes which reduce the impact of future sustained ischaemic insults. ${ }^{22}{ }^{23}$ While the majority of strokes cannot be predicted, there are several clinical settings in which an ischaemic or haemorrhagic stroke is a potential consequence of a surgical or endovascular procedure, for example, prior to treating cerebral aneurysms,${ }^{24}$ carotid endarterectomy and carotid artery stenting. ${ }^{25}$

Three studies are investigating the potential benefit of RIPreC in reduction of ischaemic stroke or vasospasm after surgical and endovascular treatment of unruptured cerebral aneurysms (NEAT - NCT03496415; RIPAT NCT02162654 ${ }^{26}$; NCT03814850; online supplemental material).

The largest of these studies (NEAT - NCT03496415), from China, aims to recruit 210 participants from multiple centres, with unruptured intracranial aneurysms, randomising them to bilateral upper limb RIPreC at $200 \mathrm{~mm} \mathrm{Hg}$ or sham RIPreC at $60 \mathrm{~mm} \mathrm{Hg}$ prior to neuroendovascular therapies (ET). The primary outcome measure of this study is the presence and volume of new DWI lesions on MRI within 72 hours of ET, while secondary outcomes include day 7 stroke severity (NIHSS score) and cerebrovascular events and modified Rankin Scale (mRS) at 30 days. Importantly, the participant, care provider, investigator and outcomes assessor are all masked to group allocation. The results from this study will indicate whether RIPreC has a potential role as an adjunct for elective aneurysm repair.

The RIPAT study (NCT02162654) ${ }^{26}$ aims to enhance our biomechanistic understanding of the potential benefits of RIPreC before treatment of unruptured aneurysms. This prospective, double-masked explorative trial in Austria, will randomise 48 individuals with asymptomatic intracranial aneurysms to RIPreC $(3 \times 5$ min cycles in the upper arm at $200 \mathrm{~mm} \mathrm{Hg})$ or sham RIPreC $(3 \times 5 \mathrm{~min}$ cycles in the upper arm at $10 \mathrm{~mm} \mathrm{Hg}$ ), while measuring inflammatory cytokines associated with the conditioning response (S100 calcium-binding protein B (S100B), 
Table 1 Summary characteristics of ongoing trials of RIC in stroke

\section{Parameter}

Study population

Timing of conditioning

\begin{tabular}{|c|c|c|c|}
\hline & & RIPerC and early RIPostC & 28 \\
\hline & & Chronic RIPostC & 16 \\
\hline & & Unspecified & 1 \\
\hline \multirow[t]{4}{*}{ Participants (N) } & & $<100$ & 24 \\
\hline & & $100-500$ & 17 \\
\hline & & $501-1000$ & 4 \\
\hline & & $>1000$ & 3 \\
\hline \multirow[t]{25}{*}{ RIC protocol } & Cuff pressure & $180 \mathrm{~mm} \mathrm{Hg}$ & 2 \\
\hline & & $200 \mathrm{~mm} \mathrm{Hg}$ & 26 \\
\hline & & $225 \mathrm{~mm} \mathrm{Hg}$ & 2 \\
\hline & & $20 \mathrm{~mm} \mathrm{Hg}>S B P$ & 4 \\
\hline & & $30 \mathrm{~mm} \mathrm{Hg}>\mathrm{SBP}$ & 2 \\
\hline & & $50 \mathrm{~mm} \mathrm{Hg}>\mathrm{SBP}$ & 1 \\
\hline & & $110 \mathrm{~mm} \mathrm{Hg}>\mathrm{SBP}$ & 1 \\
\hline & & Variable & 1 \\
\hline & & Unspecified & 9 \\
\hline & Cycle length & $3 \mathrm{~min}$ & 1 \\
\hline & & $5 \mathrm{~min}$ & 36 \\
\hline & & $10 \mathrm{~min}$ & 1 \\
\hline & & Variable & 1 \\
\hline & & Unspecified & 9 \\
\hline & Cycle frequency & 3 & 5 \\
\hline & & 4 & 16 \\
\hline & & 5 & 19 \\
\hline & & Variable & 1 \\
\hline & & Unspecified & 7 \\
\hline & Limb & Upper & 36 \\
\hline & & Lower & 5 \\
\hline & & Unspecified & 7 \\
\hline & & Unilateral & 18 \\
\hline & & Bilateral & 16 \\
\hline & & Unspecified & 14 \\
\hline \multirow[t]{3}{*}{ Study location } & & Asia & 30 \\
\hline & & Europe & 10 \\
\hline & & North America & 8 \\
\hline \multirow[t]{5}{*}{ Primary outcome measures } & Clinical & modified Rankin Scale & 6 \\
\hline & & Compliance/feasibility & 6 \\
\hline & & Adverse events & 6 \\
\hline & & Recurrent stroke & 4 \\
\hline & & Combined vascular events & 1 \\
\hline
\end{tabular}

Continued

Ischaemic stroke/TIA

Symptomatic intracranial stenosis

Aneurysmal subarachnoid haemorrhage

Unruptured cerebral aneurysm

Intracerebral haemorrhage

RIPreC

PerC and early RIPostC

No of studies

31

8

4

3

3

3

28

Chronic RIPostC 
Table 1 Continued

\begin{tabular}{|c|c|c|c|}
\hline \multicolumn{3}{|l|}{ Parameter } & \multirow{2}{*}{$\begin{array}{l}\text { No of studies } \\
1\end{array}$} \\
\hline & & All-cause mortality & \\
\hline & & NIHSS & 1 \\
\hline & & Walking speed & 1 \\
\hline & & Depression incidence & 1 \\
\hline & Blood samples & Serum biomarkers & 5 \\
\hline & & Immune profile & 2 \\
\hline & & Coagulation profile & 1 \\
\hline & Radiological & Infarct size/growth & 6 \\
\hline & & New infarcts & 2 \\
\hline & & Cerebral blood flow/collateral circulation & 3 \\
\hline & & Vasospasm & 2 \\
\hline & & Flow-mediated dilatation & 1 \\
\hline & Other & P300 event-related potential & 1 \\
\hline & & Cardiac function (echocardiogram) & 1 \\
\hline \multirow[t]{6}{*}{ Estimated study completion } & & 2019 & 11 \\
\hline & & 2020 & 10 \\
\hline & & 2021 & 8 \\
\hline & & 2022 & 5 \\
\hline & & 2023 & 0 \\
\hline & & 2024 & 3 \\
\hline
\end{tabular}

RIC, remote ischaemic conditioning; RIPerC, Remote ischaemic perconditioning; RIPostC, Remote ischaemic postconditioning; RIPreC, Remote ischaemic preconditioning; SBP, systolic blood pressure; TIA, Transient Ischaemic Attack.

Matrix Metallopeptidase 9 (MMP 9), Glial Fibrillar Acidic Protein (GFAP), Myelin Basic Protein, Neuron-Specific Enolase). Ischaemic lesions (number and volume) will be measured by DWI MRI at 12-48 hours post-treatment, with clinical outcomes (NIHSS and mRS) and neuropsychological testing measured at 6 and 12 months. A caveat to this study is that two different procedures (endovascular coiling and surgical clipping) are being used which may bias results. The current status of this trial is unknown, however, the results are of significant interest in developing our understanding of the temporal nature of the inflammatory cascade that follows RIPreC and assessing its potential impact on the development of periinterventional ischaemia.

\section{RIPerC and early RIPostC}

We identified 28 studies investigating RIPerC and early RIPostC as an adjunct to hyperacute stroke care (online supplemental material). Putative beneficial mechanisms of RIC in this hyperacute setting include increased cerebral blood flow (CBF) and perfusion of the ischaemic penumbra. ${ }^{27}$ The primary aims for many of these studies are to establish whether RIPerC and/or early RIPostC is safe and can reduce stroke severity assessed by clinical and radiological indicators. Alongside this, several studies are investigating the feasibility of hyperacute RIC alongside intravenous thrombolysis (SERICT-AIS - NCT04027621 28 ; rTPA-RIC - NCT02886390; rTPA-RIC1 - NCT03231384;
TRIPCAIS - NCT03218293) and ET (PROTECT-I NCT03915782; REVISE-2 - NCT03045055; RICE-PAC - NCT03152799). While the most trials are investigating whether RIC in ischaemic stroke, a subset of studies are investigating RIC in intracerebral haemorrhage and subarachnoid haemorrhage (online supplemental material).

The largest study of RIC in acute stroke in Europe, the Danish RESIST trial (NCT03481777) will randomise 1500 participants (baseline $\mathrm{mRS} \leq 2$ ) presenting within 4 hours of symptom onset with a prehospital putative stroke (combined Cincinattti Prehospital Stroke Scale and Postural Assessment Scale for Stroke) to RIPerC/ sham in the prehospital phase and RIPostC/sham after 6 hours in confirmed cases of acute ischaemic or haemorrhagic stroke. The active arm involves $5 \times 5$ min cycles of unilateral upper arm occlusion at $200 \mathrm{~mm} \mathrm{Hg}$ (or 35 $\mathrm{mm} \mathrm{Hg}$ above SBP if baseline SBP is $>175 \mathrm{~mm} \mathrm{Hg}$ ) while sham occlusion pressure rests at $20 \mathrm{~mm} \mathrm{Hg}$. The primary outcome measure of 3 month mRS will be supplemented with composite cardiovascular event rates at 3 months and 1 year, as well as quality of life measures and hospital bed-day use. Participants at the central site will also be randomised to RIPostC or sham RIPosC twice daily for 7 days to facilitate an assessment of the incremental effect of repeated doses of RIC. In a subset of patients, the investigators will also assess the impact of RIC on DWI infarct growth at 24 hours (in ischaemic stroke) 
and haematoma expansion on CT (in haemorrhagic stroke).

Other studies, such as RICAMIS (NCT03740971; 1800 participants) and SERIC-AIS in China (NCT03669653; 912 participants), REMOTE-CAT in Spain (NCT03375762; 572 participants) and RESCUE-BRAIN in France (NCT02189928; 200 participants), ${ }^{29}$ will be of significant value in determining the relative efficacy of RIC in hyperacute stroke in different populations. However, there are important methodological differences in the study populations, inclusion criteria, timing and duration of RIC, and endpoints that may preclude the reliability of any future meta-analysis. The key differences between the large trials of RIC in acute stroke are summarised in table 2.

\section{Chronic RIC}

We identified 16 studies investigating the use of chronic RIC in patients with stroke (online supplemental material), a large proportion of which concentrate on the secondary preventative effect in symptomatic intracranial atherosclerosis. ${ }^{15} 16$ However, a number of trials are also assessing the biological underpinnings of the protective action of RIC including platelet reactivity (NCT03635177) and CBF (PICASSO - NCT03208166, RICFAST - NCT03794947, NCT03635177, NCT02323425, NCT03589053, NCT03968068, ChiCTR1800014403). More novel studies are investigating the impact of RIC on walking (NCT04038697), skeletal muscle blood flow (NCT03635177), poststroke fatigue (RICFAST - NCT03794947) and poststroke depression (ChiCTR-OPC-17012871).

The largest study of chronic RIC is a multicentre trial in China (NCT02534545) where 3000 participants with symptomatic intracranial stenosis and a stroke or TIA (ABCD2 score $\geq 4$ ) in the previous 30 days are randomised to either RIC $(5 \times 5$ min cycles of bilateral upper limb occlusion at $200 \mathrm{~mm} \mathrm{Hg}$ once daily for 12 months) or sham RIC (same protocol at $60 \mathrm{mmHg}$ ) ${ }^{30}$ With a primary end-point of time to first recurrence of ischaemic stroke, this study may definitively answer whether chronic RIC is an effective adjunct to aggressive medical therapy in patients with symptomatic intracranial stenosis.

With several large, multicentre trials of RIC due to report their findings within the next 3 years, there will be more robust data available on the efficacy of RIC in acute and chronic stroke. However, even if the efficacy of RIC is demonstrated, several outstanding uncertainties remain which require investigation in order to optimise the delivery of RIC to the most suitable target populations. Here, we discuss which ongoing studies can address these barriers and make recommendations for future clinical trial protocols.

\section{METHOD OF DELIVERY}

\section{Dose and frequency}

The minimally effective and most effective dose and frequency of RIC is unclear. With most studies employing between 3-5 $\times 5$ minute cycles of upper limb ischaemia at $200 \mathrm{~mm} \mathrm{Hg}$ once daily, a single 'dose' of RIC can last between 30 and 50 mins. Table 1 illustrates the variation in RIC protocols used in ongoing clinical trials. By identifying the dose-response relationship between RIC and any potential clinical benefits clinicians will be better able to counsel patients with shorter cycle lengths potentially resulting in improved concordance with therapy.

In addition to the RESIST trial, investigating single and repeated RIC in acute stroke, two further studies are investigating the optimal dose and frequency of chronic RIC (NCT03105141 and ChiCTR1800014403). In the first study, NCT03105141, 600 patients with symptomatic intracranial stenosis and a recent stroke or high-risk TIA are stratified into 1 of 10 treatment groups. All the treatment groups receive automated RIC daily for 1 year with substudies assessing the effect of different cuff pressures (200 $\mathrm{mm} \mathrm{Hg}$ or $40 \mathrm{~mm} \mathrm{Hg}$ above systolic), cycle length $(4,5$ or 6 mins), cycle number $(4,5$ or 6$)$ and frequency (once daily vs twice daily) on stroke recurrence. At the same centre, ChiCTR1800014403 will randomise 273 individuals with symptomatic intracranial stenosis to either $3 \times 3$ mins or $5 \times 5$ mins cycles of RIC twice daily and compare the effect of a shorter RIC intervention on secondary prevention. Timing of RIC in Acute Stroke: The optimal timing of RIC delivery in acute stroke is unclear. Among the major trials of RIC in acute stroke there is significant heterogeneity in patient inclusion criteria and the timing of RIC (table 2). For instance, in RICAMIS (NCT03740971) only acute moderate-severe ischaemic stroke (National Institute of Health Stroke Scale, NIHSS 6-16) cases confirmed on neuroimaging and within 48 hours of symptom onset are included while, in the RESIST study (NCT03481777), RIC is commenced in a prehospital phase for patients with a presumed stroke (based on the combined Cincinnati Prehospital Stroke Scale and Postural Assessment Scale for Stroke) within 4 hours of symptom onset. A schematic of the timing and duration of RIC in the larger acute stroke trials is illustrated in figure 2. If the beneficial effects of RIC in acute stroke require early intervention, then adequately powered studies of RIC delivered in the prehospital phase are essential to establish its efficacy.

Duration: It remains unclear whether there is a ceiling effect of repeated, daily RIC; if RIC is still efficacious when delivered on a less frequent basis, this may increase uptake and concordance. In chronic stroke, RIC has been used daily for up to 300 days. ${ }^{15}$ Meng et al reported on a proof-of-concept study of twice daily bilateral upper limb RIC for 300 days in patients with symptomatic intracranial stenosis. Those in the treated arm $(n=38)$ experienced significant reductions in stroke recurrence after 90 and 300 days of RIC compared with controls treated with medical therapy alone $(\mathrm{n}=30) .{ }^{15}$ The Kaplan-Meier analysis of stroke recurrence indicates deviation of RIC group from controls after around 60 consecutive days of RIC, however, it is unclear whether a shorter duration of chronic RIC (eg, 60 days) would be sufficient to 


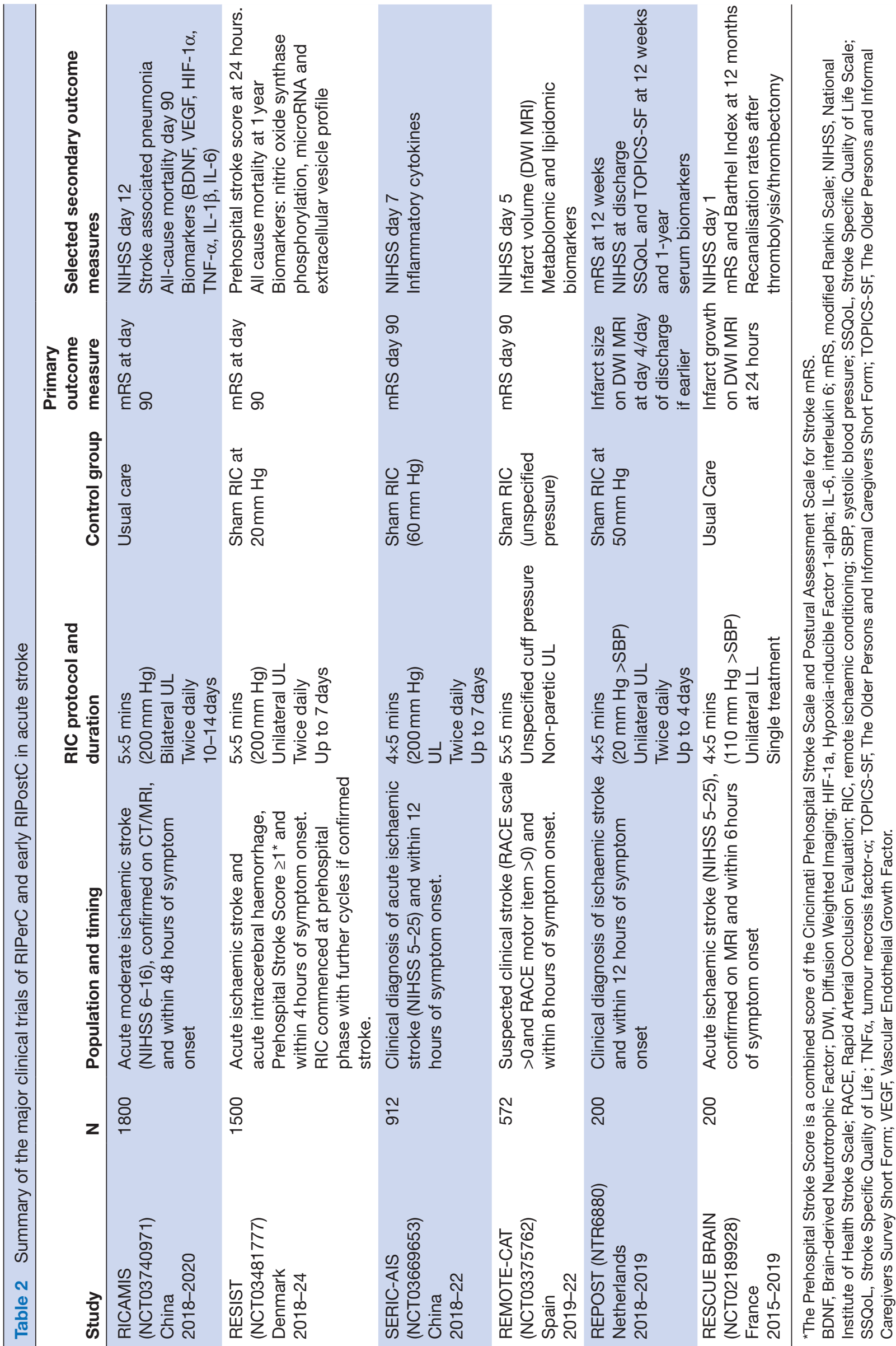




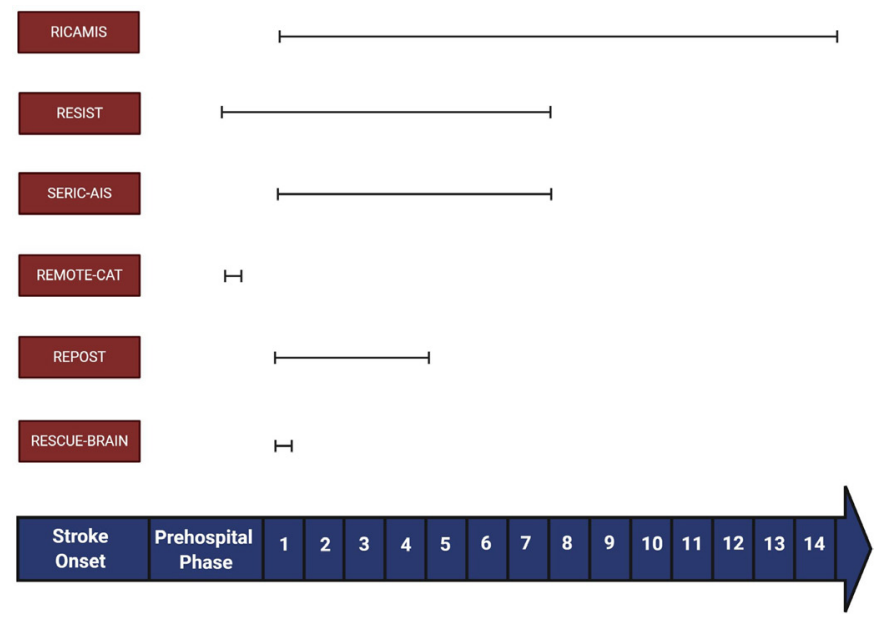

Figure 2 A schematic of RIC timing and duration in major trials of acute stroke. RIC, remote ischaemic conditioning.

sustain a clinically beneficial effect over the long term. The maximum follow-up time in current RIC studies is 12 months, which may be suboptimal given the follow-up times required to prove effectiveness in secondary prevention trials of antiplatelets and statins. ${ }^{31} 32$ Taken together, this could help establish an agreed protocol of either a single course of RIC, periodic RIC or lifelong RIC. Both the use of RIC for stroke prevention, potentially lifelong, and long term trial follow-up, are significant investments for patients and trialists, respectively. The discovery of validated biomarkers for vascular protection could shorten follow-up times and identify patients responding to treatment, negating the need for unnecessary long term treatment.

\section{Unilateral versus bilateral RIC}

While bilateral RIC may theoretically produce a larger ischaemic stimulus, no study is currently comparing the clinical impact of unilateral vs bilateral RIC. Unilateral RIC may be more convenient for individuals with hemiparesis and may allow concurrent completion of daily tasks during therapy. Furthermore, in the context of hyperacute stroke, the use of unilateral RIC enables the other arm to be used for venous access and intravenous thrombolysis.

For unilateral RIC, it is unclear if the marginal gain from repeated conditioning in the same limb diminishes over time. As such, it would be helpful to establish whether alternating the site of delivery has a role in maintaining the conditioning stimulus over time. Furthermore, it is unclear whether the use of unilateral RIC in the paretic arm yields the same conditioning response as RIC in the non-paretic arm; experimentally, nerve transection has been shown to block the effect of preconditioning, ${ }^{33}$ however, this has not been established for organic central lesions.

\section{Upper limb versus lower limb RIC}

While lower limb RIC has the theoretical potential to induce a larger ischaemic stimulus, a study of RIC in healthy volunteers showed no significant differences in the production of nitrite, a potential signalling mediator of the conditioning response, after upper limb or lower limb RIC in healthy volunteers. ${ }^{34}$ However, as discussed, given the conditioning response likely involves multiple signalling cascades that occur over different time frames, there is insufficient evidence to conclude that upper limb and lower limb RIC are equipotent. We found only five studies investigating lower limb RIC (RESCUE-BRAIN (NCT02189928), ${ }^{29}$ PreLIMBS (NCT02411266), RIPC-SAH (NCT02381522), NCT04039399, NCT04038697). The preponderance of interventions using upper limb RIC may reflect increased practicality for both patients and carers. A qualitative analysis of patient experience with both upper and lower limb RIC may reveal key barriers to longer-term compliance that can help investigators optimise intervention strategies.

\section{Safety and tolerability}

Multiple clinical trials suggest that RIC is safe,,$^{20} 21$ even when performed twice daily on both arms for 300 days, ${ }^{15}$ including in the elderly. ${ }^{16}$ However, relatively few studies of chronic RIC have reported on compliance, therefore, it is difficult to ascertain whether stroke patients are able to carry out RIC for sustained periods of time. In Meng et $a l,{ }^{15} 51$ patients with symptomatic intracranial stenosis were randomised to receive twice daily bilateral arm conditioning for 300 consecutive days but only 38 (74.5\%) completed the study. Long-term data on larger cohorts will help inform clinicians on likely compliance rates.

A small number of side effects have been reported including skin petechiae, ${ }^{16}$ but no studies have indicated increased risks of venous thrombosis or rhabdomyolysis. It is unclear if chronic RIC affects sensory or motor function of the conditioned limbs; studies that include these detailed clinical and electrophysiological assessments as outcome measures should help fill this knowledge gap.

A consistent exclusion criterion for several clinical trials of RIC in stroke has been known peripheral vascular disease or venous thromboembolism affecting the conditioned limb. While there is a theoretical risk of harm in these patients, the fact that prior studies have not highlighted incident risk of these complications, and that studies are investigating upper limb RIC in patients with known lower limb peripheral arterial disease, ${ }^{35}$ should provide some reassurance to clinicians.

\section{RIC and ET}

The usefulness of RIC alongside ET for large vessel occlusion (LVO) has attracted much research interest. Numerous questions arise. The first is whether RIC can be delivered parallel to ET without causing significant treatment delays. The second is whether, RIC performed alongside recanalisation of a culprit vessel may influence cerebral autoregulation in a manner that increases the risk of reperfusion injury or haemorrhagic transformation. Hypertension and impaired cerebral autoregulation 
often already accompany acute ischaemic stroke. ${ }^{36} 37$ The third is whether the effect of general anaesthetic diminishes the effect of RIC; as previous studies have indicated, the use of propofol inhibits the ischaemic conditioning response. ${ }^{38}$

\section{Manual versus automated RIC}

Automated RIC devices are widely available. While RIC has been marketed as a potentially low-cost, non-invasive intervention for vascular diseases, the costs of obtaining multiple devices, cuff and battery replacements, may prove to be prohibitively expensive in low and middle income countries where the rates of cerebrovascular disease remain high. ${ }^{39}$ As such, studies which deploy manual RIC (with sphygmomanometers and digital timers) will help develop the global translatability of RIC.

Three studies plan to compare the effect of RIC in those undergoing ET (REVISE-2 - NCT03045055, PROTECT-I - NCT03915782, RICE-PAC - NCT03152799) (online supplemental material). As well as revealing efficacy, these will highlight the practical challenges of delivering RIC to patients in a time-sensitive manner.

\section{Target populations}

Intracranial stenosis: The majority of studies of chronic RIC have been performed in individuals with significant intracranial stenosis. This is an important population to study for two reasons: high stroke recurrence rates in this population mean a lower sample size is needed to detect a significant treatment effects, and they are a subgroup where significant improvements in care can potentially be made. However, while intracranial atherosclerosis contributes to ischaemic stroke in a large proportion of individuals globally, it is a less common mechanism of stroke in European and North American populations where extracranial carotid disease, cardioembolic stroke and small vessel disease are more common. ${ }^{40}{ }^{41}$ As such, studies need to extend to individuals without intracranial stenosis to establish the utility of RIC in the wider setting of secondary stroke prevention. Furthermore, most studies of chronic RIC in ischaemic stroke have been performed in East Asia; while there is no identifiable reason why the response to RIC would be different in a different population, it is important for similar studies to be undertaken outside of Asia to identify whether the chronic RIC is both tolerated and beneficial in a wider global setting.

The influence of comorbidities and medications: It is recognised that the impact of RIC may diminish with age. ${ }^{42}$ However, Meng et $a l,{ }^{16}$ demonstrated that RIC may reduce stroke recurrence in those over 80 thereby suggesting that ageing alone may not suppress the beneficial effects of RIC.

Diabetes mellitus is a common risk factor for ischaemic stroke, however, evidence from both animal models and clinical studies suggest that diabetes, particularly diabetic neuropathy, may abolish the protective effect of RIC. ${ }^{43}$ For instance, a recent study of chronic, daily RIC in patients with diabetes with peripheral arterial disease failed to show any positive impact on vascular or neuronal function $^{35}$; potentially linked to impairment of the neurogenic pathways thought to potentiate the action of RIC. ${ }^{43}$ The theoretical risk of compounding nerve damage with repeated limb compression in chronic RIC may also be a reason for excluding such patients from trials, and their ultimate under-representation in the literature. Subgroup analyses of larger randomised controlled trials or pooled datasets may help determine if the presence of diabetes truly impacts on the clinical effects of RIC.

Animal models also suggest that the conditioning response is diminished by the presence of comorbidities such as hypercholesterolaemia, hypertension and obesity. ${ }^{42}$ However, this has not yet been confirmed in clinical studies of RIC in stroke. Similarly, several medications that are commonly prescribed in individuals with cardiovascular disease including nitrates, atorvastatin and nicorandil may activate similar intracellular signalling cascades to RIC. ${ }^{44}$ However, clinical data are lacking with respect to understanding whether this would potentiate or abrogate the effects of RIC in patients taking these medications.

\section{Cardioembolic stroke}

Studies of RIC in ischaemic stroke typically exclude individuals with known cardioembolism from causes such as atrial fibrillation or flutter. In these patients, stroke often results from the sudden migration of thrombus from the heart to the cerebral arteries, a pathway that RIC may not obviously affect at first glance. However, the impact of RIC on secondary prevention of cardioembolic stroke is of interest for several reasons. First, the seminal studies investigating the protective effect of ischaemic preconditioning were performed in animal models where ischaemia was precipitated acutely, ${ }^{22}{ }^{23}$ a mode of infarction more akin to cardio-embolism than atherosclerotic disease. It may be that slow atheromatous progression and intracranial stenosis leads to a degree of endogenous pre-conditioning that reduces the marginal impact of RIC compared with those with cardio-embolic stroke. Consistent with this, previous studies have indicated that the volume of ischaemia resulting from cardioembolism is larger than that due to a similar sized clot from the carotid/intracranial circulation. ${ }^{45}$ Furthermore, the most common cause of cardio-embolism, atrial fibrillation, develops predominantly in later life and in the context of cardiovascular risk factors such as hypertension and diabetes mellitus. As such, many patients with atrial fibrillation have associated endothelial dysfunction, ${ }^{46}$ another pathway potentially impacted by RIC. ${ }^{47}$

\section{Small vessel ischaemic disease}

Small vessel ischaemic disease (SVID) is a common comorbidity and complication associated with stroke and is a precursor for the development of vascular cognitive impairment (VCI) and vascular dementia. The role of RIC in SVID has attracted interest due its potential effects on 
CBF and inflammatory markers. In SVID, hypoperfusion due to microangiopathy can precipitate neurodegeneration through disruption of the blood-brain barrier and neuroinflammation. ${ }^{48}$ Declines in cognitive function have also been associated with reduced microvascular perfusion. ${ }^{49}$ Small pilot studies of patients with established SVID or those with VCI have shown that RIC applied twice daily ( $5 \times 5$ min cycles) for between 6 and 12 months can result in improvements in $\mathrm{CBF}$ velocities, reductions volume of white matter lesions, and variable improvements in domains of cognitive function. ${ }^{50-52}$ Investigations are currently under way to evaluate whether once daily application, for a shorter duration of 1 month has similar effects with greater treatment adherence. ${ }^{53}$

\section{RIC and exercise}

Experimental and clinical evidence suggests shared biological mechanisms for the effects of RIC and exercise, including increased heat shock proteins and increased endothelial nitric oxide signalling. ${ }^{54}$ It is unclear whether there is any synergistic effect of RIC when performed in addition to regular exercise, and no current studies evaluating this in chronic stroke. While exercise confers many physiological benefits in stroke patients, ${ }^{55}$ numerous barriers to exercise poststroke exist including depression, fatigue and physical disability. As such, if there are physiological effects of RIC that mirror exercise, then this may be a useful long-term ancillary treatment when patients are physically unable to exercise.

\section{Underlying mechanism and biomarkers}

Applying an external pressure to occlude blood flow to a limb initiates transmission of a signal that mediates the RIC response. Humoral, neurogenic and immune mediated pathways have been proposed as transmission and end organ effector mechanisms. ${ }^{9}$ After the transmission of the conditioning stimulus, an array of possible biochemical and physiological changes may contribute to clinically meaningful outcomes in stroke patients including increased $\mathrm{CBF}^{15}$ angiogenesis and increased collateral circulation, ${ }^{27}$ antiplatelet effects, ${ }^{56}{ }^{57}$ synaptogenesis, ${ }^{58}$ immune regulation, mitochondrial function and autophagy regulation, ${ }^{47}$ (figure 3 ). The latency of these biological mediators and effectors may vary and are likely to underpin differences in effects of acute and chronic RIC. ${ }^{9}$ Establishing the underlying mechanisms and final effectors of RIC will prove essential in optimising intervention development, trial design and establishing its efficacy and safety in wider clinical practice. Furthermore, by characterising the intracellular pathways associated with the beneficial effects of RIC, appropriate drug targets may be identified, leading the way towards pharmacological conditioning. ${ }^{59}$

For subarachnoid haemorrhage, the putative biological mechanism for any potential benefit of RIC are not well characterised but may involve reducing vasospasm. ${ }^{60} \mathrm{In}$ intracerebral haemorrhage, there are pathophysiological consequences that are shared with ischaemic stroke

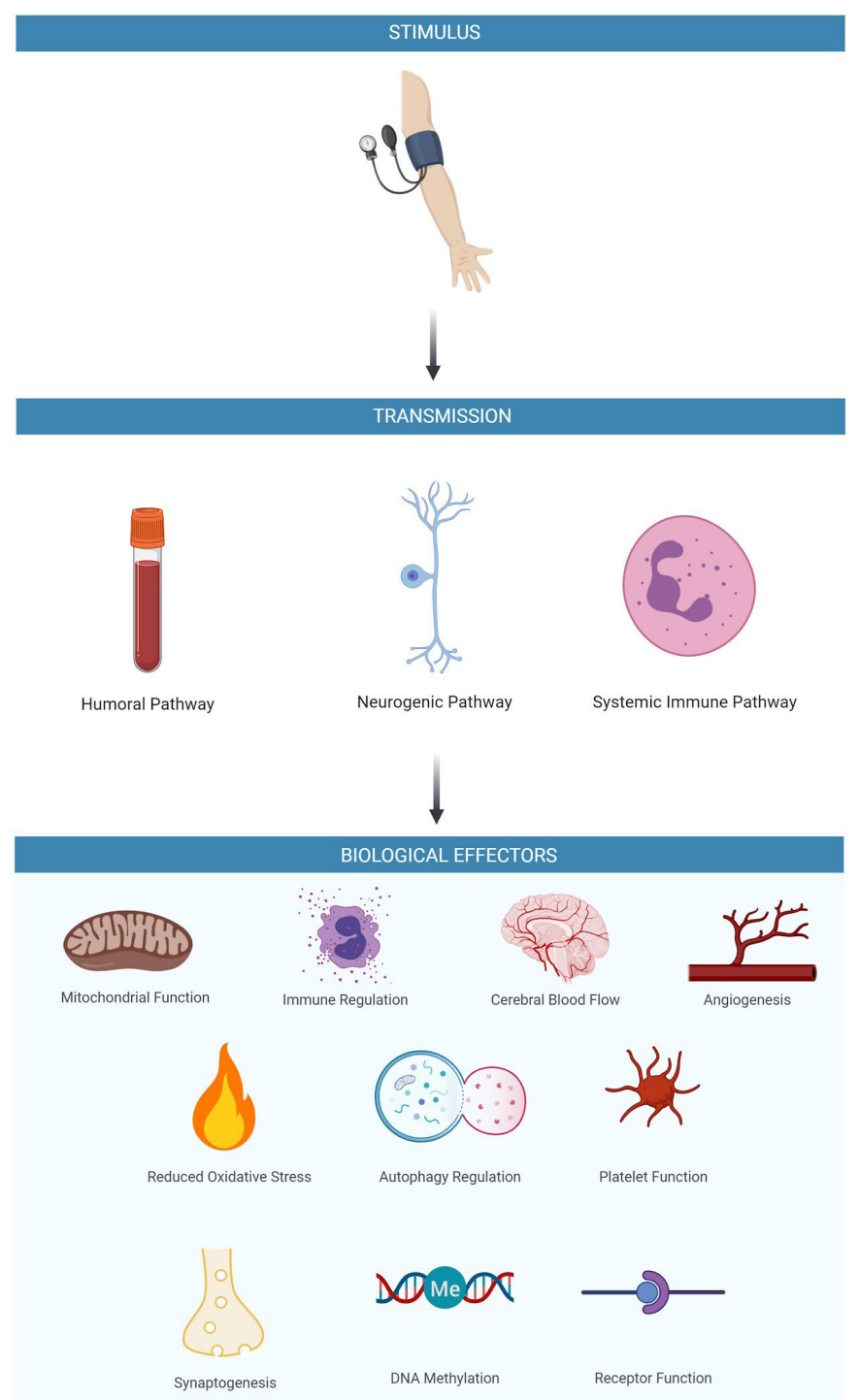

Figure 3 Proposed mechanism of action of remote ischaemic conditioning in stroke.

including endothelial dysfunction, impaired cerebral autoregulation, a proinflammatory mileu and mitochondrial dysfunction ${ }^{61}$; these have all been proposed as therapeutic targets of RIC in ischaemic stroke, and therefore, may prove to be of significance in intracerebral haemorrhage.

\section{Circulating biomarkers}

Previous studies have indicated potential serum signalling mediators of RIC including heat shock proteins, tumor necrosis factor (TNF)- $\alpha$ and interleukin 6 (IL-6). ${ }^{9}$ However, no established circulating biomarkers have been shown to be reliably correlate with the degree of conditioning response or clinical outcomes. Identifying a serum biomarker would be advantageous as it could offer a minimally invasive measure to: (1) establish which patients are responding to the treatment and (2) optimise the dose, frequency and duration of RIC treatment on an individualised basis. Several ongoing studies are investigating candidate circulating biomarkers including 


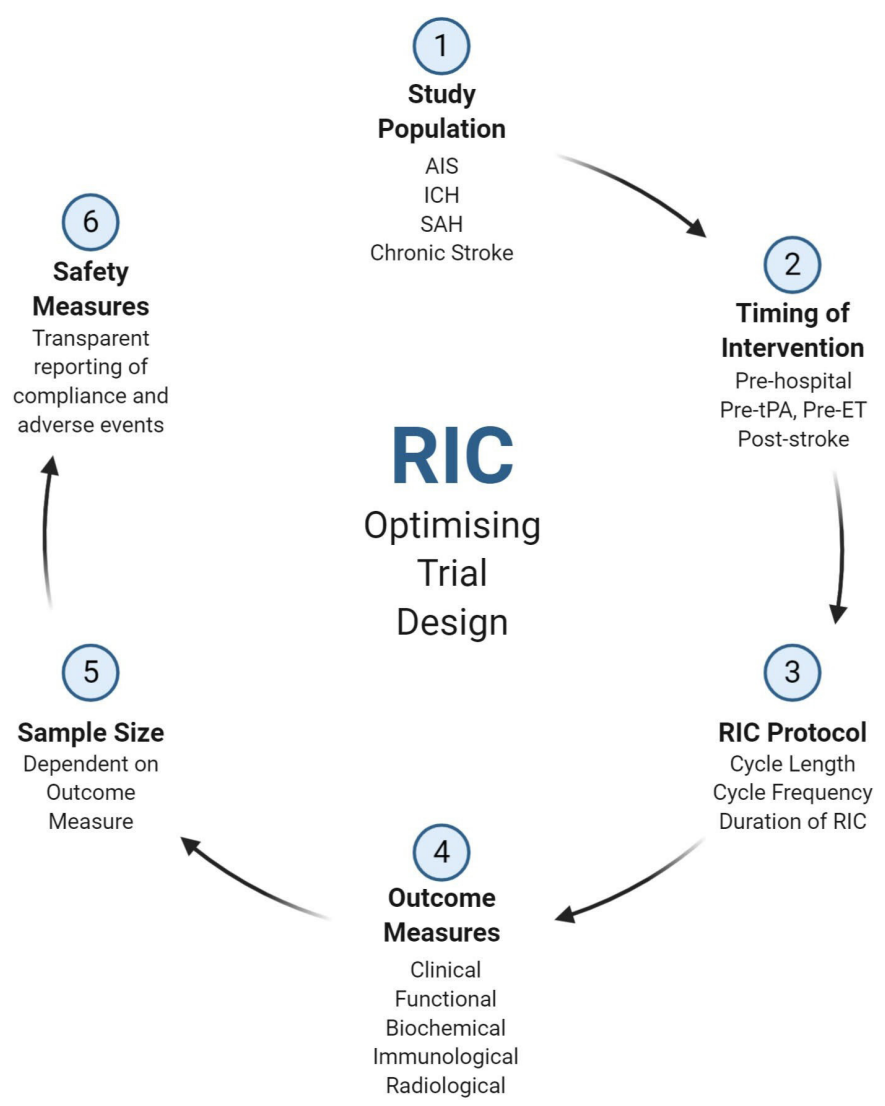

Figure 4 Optimising remote ischaemic conditioning (RIC) trial design. AIS, Acute Ischaemic Stroke; ET, endovascular therapy; ICH, Intracerebral haemorrhage; SAH, Subarachnoid Haemorrhage; tPA, Tissue Plasminogen Sctivator.

NCT02323425 (VEGF and bFGF), RESIST - NCT03481777 (eryNOS3 phosphorylation, microRNA and extracellular vesicle profile), NCT03546309 (S100-A4, MMP-9, bFGF, PDGF, VEGF) and NCT03335111 (IL-4, IL-6, TNF) (online supplemental material). Future studies of RIC in stroke should include focused analyses of candidate biomarkers, identified from experimental animal and human models, at different time points before, during and after both acute and chronic RIC. It is also important to validate which biomarkers are associated with the transmission of the ischaemic signal and which are associated with the biological effector response and clinical outcomes.

\section{Radiological correlates}

Animal models, ${ }^{27}$ and previous clinical studies, ${ }^{15}$ indicate that RIC can increase CBF as well as increasing the dynamic range of cerebral autoregulation in healthy volunteers. ${ }^{37}$ In the setting of acute ischaemic stroke, RIPerC may increase CBF (directly or via collaterals) to salvage the ischaemic penumbra. ${ }^{27}$ Several studies are investigating the effect of RIPerC on infarct volume and clinical outcomes acutely (online supplemental material), however, few trials use non-invasive measures such as Transcranial Doppler (TCD) to characterise the impact of RIC on blood vessel diameter and flow.
Several studies investigating chronic RIC are measuring cerebral and skeletal muscle blood flow using a range of modalities including TCD, Arterial Spin Labelling and MR angiography (NCT03635177, NCT02323425 and NCT04038697). If increased cerebral or skeletal muscle blood flow is associated with positive clinical outcomes in the larger studies of chronic RIC then non-invasive assessments of blood flow could be used to establish response to RIC on a personalised level.

\section{Economic analysis}

The costs of automated conditioning devices, device maintenance and safety monitoring need to be factored into a cost-benefit analysis of RIC in stroke. Furthermore, additional care staff may be required to administer RIC to individuals who are unable to independently apply the device.

\section{Recommendations for future trial design}

Given the multitude of ongoing studies for RIC in stroke, a collaborative effort is required to synthesise the array of results into meaningful outcomes for stroke patients. Figure 4 represents a proposed schema for future clinical trial design, identifying six key areas which future studies should focus their attention in order to address the remaining uncertainties of RIC in stroke.

\section{CONCLUSIONS}

RIC has been shown to be a promising therapy in animal models of stroke while smaller clinical studies have indicated its safety and feasibility in humans. However, there are several remaining questions before results of these studies can be translated into widespread clinical practice. Ongoing clinical trials will inform whether RIC is effective in the acute and chronic setting of stroke. However, there is an urgent need to concurrently investigate which patient groups respond best to RIC, identify the optimal dose and duration of therapy, and to establish biological and radiological biomarkers of the conditioning response before RIC can advance into routine clinical practice.

\section{Twitter Ali Ali @dralinali}

Contributors AA conceived the review topic and supervised all aspects of data collection, analysis and report writing. BM and SB undertook data collection and analysis. All authors contributed to data analysis and manuscript writing.

Funding This study was funded by NIHR Sheffield Biomedical Research Centre (BRC).

Competing interests None declared.

Patient consent for publication Not required.

Provenance and peer review Not commissioned; externally peer reviewed.

Open access This is an open access article distributed in accordance with the Creative Commons Attribution Non Commercial (CC BY-NC 4.0) license, which permits others to distribute, remix, adapt, build upon this work non-commercially, and license their derivative works on different terms, provided the original work is properly cited, appropriate credit is given, any changes made indicated, and the use is non-commercial. See: http://creativecommons.org/licenses/by-nc/4.0/.

ORCID iD

Ali Ali http://orcid.org/0000-0001-6339-4076 


\section{REFERENCES}

1 Heusch G, Bøtker HE, Przyklenk K, et al. Remote ischemic conditioning. J Am Coll Cardiol 2015;65:177-95.

2 Murry CE, Jennings RB, Reimer KA. Preconditioning with ischemia: a delay of lethal cell injury in ischemic myocardium. Circulation 1986;74:1124-36.

3 Przyklenk K, Bauer B, Ovize M, et al. Regional ischemic 'preconditioning' protects remote virgin myocardium from subsequent sustained coronary occlusion. Circulation 1993;87:893-9.

4 Gho BC, Schoemaker RG, van den Doel MA, et al. Myocardial protection by brief ischemia in noncardiac tissue. Circulation 1996;94:2193-200.

5 Jensen HA, Loukogeorgakis S, Yannopoulos F, et al. Remote ischemic preconditioning protects the brain against injury after hypothermic circulatory arrest. Circulation 2011;123:714-21.

6 Tapuria N, Kumar Y, Habib MM, et al. Remote ischemic preconditioning: a novel protective method from ischemia reperfusion injury--a review. J Surg Res 2008;150:304-30.

7 Wever KE, Warlé MC, Wagener FA, et al. Remote ischaemic preconditioning by brief hind limb ischaemia protects against renal ischaemia-reperfusion injury: the role of adenosine. Nephrol Dial Transplant 2011;26:3108-17.

8 Candilio L, Malik A, Hausenloy DJ. Protection of organs other than the heart by remote ischemic conditioning. J Cardiovasc Med 2013;14:193-205.

9 Zhou G, Li MH, Tudor G, et al. Remote ischemic conditioning in cerebral diseases and neurointerventional procedures: recent research progress. Front Neurol 2018;9:339.

10 Doeppner TR, Zechmeister B, Kaltwasser B, et al. Very delayed remote ischemic post-conditioning induces sustained neurological recovery by mechanisms involving enhanced angioneurogenesis and peripheral immunosuppression reversal. Front Cell Neurosci 2018;12:383.

11 Khan MB, Hafez S, Hoda MN, et al. Chronic remote ischemic conditioning is cerebroprotective and induces vascular remodeling in a VCID model. Trans/ Stroke Res 2018;9:51-63.

12 Durand MJ, Boerger TF, Nguyen JN, et al. Two weeks of ischemic conditioning improves walking speed and reduces neuromuscular fatigability in chronic stroke survivors. J Appl Physiol 2019;126:755-63.

13 England TJ, Hedstrom A, O'Sullivan S, et al. RECAST (remote ischemic conditioning after stroke trial): a pilot randomized placebo controlled phase II trial in acute ischemic stroke. Stroke 2017;48:1412-+.

14 Hougaard KD, Hjort N, Zeidler D, et al. Remote ischemic perconditioning as an adjunct therapy to thrombolysis in patients with acute ischemic stroke: a randomized trial. Stroke 2014;45:159-67.

15 Meng R, Asmaro K, Meng L, et al. Upper limb ischemic preconditioning prevents recurrent stroke in intracranial arterial stenosis. Neurology 2012;79:1853-61.

16 Meng R, Ding Y, Asmaro K, et al. Ischemic conditioning is safe and effective for octo- and nonagenarians in stroke prevention and treatment. Neurotherapeutics 2015;12:667-77.

17 Zhao W, Che R, Li S, et al. Remote ischemic conditioning for acute stroke patients treated with thrombectomy. Ann Clin Transl Neurol 2018;5:850-6.

18 Zhao W, Meng R, Ma C, et al. Safety and efficacy of remote ischemic preconditioning in patients with severe carotid artery stenosis before carotid artery stenting: a proof-of-concept, randomized controlled trial. Circulation 2017;135:1325-+.

19 England TJ, Hedstrom A, O'Sullivan SE, et al. Remote ischemic conditioning after stroke trial 2: a phase Ilb randomized controlled trial in hyperacute stroke. J Am Heart Assoc 2019;8:e013572.

20 Landman TRJ, Schoon Y, Warle MC. Remote ischemic conditioning as an additional treatment for acute ischemic stroke the preclinical and clinical evidence. Stroke 2019;50:1934-9.

21 Zhao W, Li S, Ren C, et al. Remote ischemic conditioning for stroke: clinical data, challenges, and future directions. Ann Clin Trans/ Neurol 2019;6:186-96.

22 Murry CE, Jennings RB, Reimer KA. Preconditioning with ischemia: a delay of lethal cell injury in ischemic myocardium. Circulation 1986;74:1124-36.

23 Przyklenk K, Bauer B, Ovize M, et al. Regional ischemic 'preconditioning' protects remote virgin myocardium from subsequent sustained coronary occlusion. Circulation 1993;87:893-9.

24 Brinjikji W, Murad MH, Lanzino G, et al. Endovascular treatment of intracranial aneurysms with flow diverters: a meta-analysis. Stroke 2013;44:442-7.
25 Muller MD, von Felten S, Algra A, et al. Immediate and delayed procedural stroke or death in stenting versus endarterectomy for symptomatic carotid stenosis. J Vasc Surg 2019;69:968-9.

26 Tülü S, Mulino M, Pinggera D, et al. Remote ischemic preconditioning in the prevention of ischemic brain damage during intracranial aneurysm treatment (RIPAT): study protocol for a randomized controlled trial. Trials 2015;16:594.

27 Kitagawa K, Saitoh M, Ishizuka K, et al. Remote limb ischemic conditioning during cerebral ischemia reduces infarct size through enhanced collateral circulation in murine focal cerebral ischemia. $J$ Stroke Cerebrovasc Dis 2018;27:831-8.

28 He Y-D, Guo Z-N, Qin C, et al. Remote ischemic conditioning combined with intravenous thrombolysis for acute ischemic stroke. Ann Clin Transl Neurol 2020;7:972-9.

29 Pico F, Rosso C, Meseguer E, et al. A multicenter, randomized trial on neuroprotection with remote iSchemic per-conditioning during acute iSchemic stroke: the remote iSchemic conditioning in acute brain infarction study protocol. Int J Stroke 2016;11:938-43.

30 Hou C, Duan J, Luo Y, et al. Remote limb ischemic conditioning treatment for intracranial atherosclerotic stenosis patients. Int $J$ Stroke 2016;11:831-8.

31 Sacco RL, Diener H-C, Yusuf S, et al. Aspirin and extended-release dipyridamole versus clopidogrel for recurrent stroke. N Engl J Med 2008;359:1238-51.

32 , Halkes PHA, van Gijn J, et al, ESPRIT Study Group. Aspirin plus dipyridamole versus aspirin alone after cerebral ischaemia of arterial origin (ESPRIT): randomised controlled trial. Lancet 2006;367:1665-73

33 Steensrud T, Li J, Dai X, et al. Pretreatment with the nitric oxide donor SNAP or nerve transection blocks humoral preconditioning by remote limb ischemia or intra-arterial adenosine. Am J Physiol Heart Circ Physiol 2010;299:H1598-603.

34 Dezfulian C, Taft M, Corey C, et al. Biochemical signaling by remote ischemic conditioning of the arm versus thigh: is one raise of the cuff enough? Redox Biol 2017;12:491-8.

35 Hansen CS, Jørgensen ME, Fleischer J, et al. Efficacy of long-term remote ischemic conditioning on vascular and neuronal function in type 2 diabetes patients with peripheral arterial disease. J Am Heart Assoc 2019;8:e011779.

36 Chi N-F, Hu H-H, Wang C-Y, et al. Dynamic cerebral autoregulation is an independent functional outcome predictor of mild acute ischemic stroke. Stroke 2018;49:2605-11.

37 Guo Z-N, Guo W-T, Liu J, et al. Changes in cerebral autoregulation and blood biomarkers after remote ischemic preconditioning. Neurology 2019;93:e8-19.

38 Kottenberg E, Thielmann M, Bergmann L, et al. Protection by remote ischemic preconditioning during coronary artery bypass graft surgery with isoflurane but not propofol - a clinical trial. Acta Anaesthesiol Scand 2012;56:30-8.

39 Feigin VL, Forouzanfar MH, Krishnamurthi R, et al. Global and regional burden of stroke during 1990-2010: findings from the global burden of disease study 2010. Lancet 2014;383:245-55.

40 Kolominsky-Rabas PL, Weber M, Gefeller O, et al. Epidemiology of ischemic stroke subtypes according to TOAST criteria: incidence, recurrence, and long-term survival in ischemic stroke subtypes: a population-based study. Stroke 2001;32:2735-40.

41 White $\mathrm{H}$, Boden-Albala B, Wang $\mathrm{C}$, et al. Ischemic stroke subtype incidence among whites, blacks, and Hispanics: the Northern Manhattan study. Circulation 2005;111:1327-31.

42 McCafferty K, Forbes S, Thiemermann C, et al. The challenge of translating ischemic conditioning from animal models to humans: the role of comorbidities. Dis Model Mech 2014;7:1321-33.

43 Tyagi S, Singh N, Virdi JK, et al. Diabetes abolish cardioprotective effects of remote ischemic conditioning: evidences and possible mechanisms. J Physiol Biochem 2019;75:19-28.

44 Sivaraman V, Yellon DM. Pharmacologic therapy that simulates conditioning for cardiac ischemic/reperfusion injury. $J$ Cardiovasc Pharmacol Ther 2014;19:83-96.

45 Weih M, Kallenberg K, Bergk A, et al. Attenuated stroke severity after prodromal TIA: a role for ischemic tolerance in the brain? Stroke 1999;30:1851-4.

46 Guazzi M, Arena R. Endothelial dysfunction and pathophysiological correlates in atrial fibrillation. Heart 2009;95:102-6.

47 You J, Feng L, Bao L, et al. Potential applications of remote limb ischemic conditioning for chronic cerebral circulation insufficiency. Front Neurol 2019;10:467.

48 Malojcic B, Giannakopoulos P, Sorond FA, et al. Ultrasound and dynamic functional imaging in vascular cognitive impairment and Alzheimer's disease. BMC Med 2017;15:27. 
49 Zhang CE, Wong SM, Uiterwijk R, et al. Intravoxel incoherent motion imaging in small vessel disease: microstructural integrity and microvascular perfusion related to cognition. Stroke 2017;48:658-63.

$50 \mathrm{Mi}$, Yu F, Ji X, et al. The interventional effect of remote ischemic preconditioning on cerebral small vessel disease: a pilot randomized clinical trial. Eur Neurol 2016;76:28-34.

51 Wang $\mathrm{Y}$, Meng $\mathrm{R}$, Song $\mathrm{H}$, et al. Remote ischemic conditioning may improve outcomes of patients with cerebral small-vessel disease. Stroke 2017;48:3064-72.

52 Liao Z, Bu Y, Li M, et al. Remote ischemic conditioning improves cognition in patients with subcortical ischemic vascular dementia. BMC Neurol 2019;19:206.

53 Ganesh A, Barber P, Black SE, et al. Trial of remote ischaemic preconditioning in vascular cognitive impairment (TRIC-VCI): protocol. BMJ Open 2020;10:e040466.

54 Zhao W, Li S, Ren C, et al. Chronic Remote Ischemic Conditioning May Mimic Regular Exercise:Perspective from Clinical Studies. Aging Dis 2018;9:165-71.

55 Wang C, Redgrave J, Shafizadeh M, et al. Aerobic exercise interventions reduce blood pressure in patients after stroke or transient ischaemic attack: a systematic review and meta-analysis. Br J Sports Med 2019;53:1515-25.

56 Alpuche J, Quírino L, Sánchez-Vega JT, et al. The role of platelets in ischemic conditioning. Cond Med 2018;1:313-8.

57 Linden MD, Whittaker P, Frelinger AL, et al. Preconditioning ischemia attenuates molecular indices of platelet activation-aggregation. $J$ Thromb Haemost 2006;4:2670-7.

58 Wang Y, Zhang Z, Zhang L, et al. RLIPostC protects against cerebral ischemia through improved synaptogenesis in rats. Brain Inj 2018;32:1429-36.

59 Esposito E, Desai R, Ji X. Pharmacologic pre- and postconditioning for stroke: basic mechanisms and translational opportunity. Brain Circulation 2015;1:104-13.

60 Koch S, Katsnelson M, Dong C, et al. Remote ischemic limb preconditioning after subarachnoid hemorrhage: a phase lb study of safety and feasibility. Stroke 2011;42:1387-91.

61 Aronowski J, Zhao X. Molecular pathophysiology of cerebral hemorrhage. Stroke 2011;42:1781-6. 